\title{
Discovery and Synthesis of a Novel Series of Liver X Receptor Antagonists
}

\author{
Siyun Nian, ${ }^{a}$ Xia Gan, ${ }^{a}$ Xiangduan Tan, ${ }^{b}$ Zhenpeng Yu, ${ }^{c}$ Panfeng Wang ${ }^{c}$ Xing Chen, ${ }^{a}$ and \\ Guoping Wang*,a,c \\ ${ }^{a}$ Shanghai Institute of Pharmaceutical Industry, China State Institute of Pharmaceutical Industry; Shanghai 200437, \\ P.R. China: ${ }^{b}$ College of Pharmacy, Guilin Medical University; Guilin, Guangxi 541004, P. R. China: and ${ }^{c}$ Shanghai \\ Shyndec Pharmaceutical Co., Ltd.; Shanghai 200437, P.R. China. \\ Received March 21, 2015; accepted May 17, 2015; advance publication released online June 11, 2015
}

Fourteen novel compounds were prepared and their antagonistic activities against liver $X$ receptors (LXR) $\alpha / \beta$ were tested in vitro. Compound 26 had an $\mathrm{IC}_{50}$ value of $6.4 \mu \mathrm{M}$ against $\mathrm{LXR} \alpha$ and an $\mathrm{IC}_{50}$ value of $5.6 \mu \mathrm{M}$ against LXR $\beta$. Docking studies and the results of structure-activity relationships support the further development of this chemical series as $L X R \alpha / \beta$ antagonists.

Key words liver $\mathrm{X}$ receptor $\alpha$; liver $\mathrm{X}$ receptor $\beta$; antagonistic activity

Nuclear receptors (NRs) are ligand-activated transcription factors that coordinate gene expression in response to the modulation of metabolism, development, proliferation, and inflammation. ${ }^{1,2}$ Liver X receptors (LXRs) belonging to the NR superfamily are activated by specific oxidized forms of cholesterol and intermediate products of the cholesterol biosynthetic pathway. ${ }^{1,3,4)}$ There are two LXR isoforms in mammals, termed $\operatorname{LXR} \alpha$ and $\operatorname{LXR} \beta . \operatorname{LXR} \alpha$ is abundantly expressed mainly in the liver, intestine, kidney, spleen, and adipose tissue, whereas $\operatorname{LXR} \beta$ is more ubiquitously expressed, with particularly high levels in the developing brain. ${ }^{5-8)}$ Both isoforms share almost $80 \%$ homology of their amino acid sequences in their DNA-binding domain and ligand-binding domain. ${ }^{5,7)}$

The LXR consists of four domains: N-terminal ligandindependent activation function domain (AF-1); DNA-binding domain (DBD); hydrophobic ligand-binding domain (LBD); and $\mathrm{C}$-terminal ligand-dependent transactivation sequence (AF-2). ${ }^{5,9,10)}$ By forming heterodimers with retinoid $\mathrm{X}$ receptors (RXRs), LXRs bind to LXR response elements (LXREs) in the promoter or enhancer elements of LXR target genes. The activation of LXR-RXR heterodimers not only induces the expression of a variety of target genes (CYP7A, ABCA1, SREBP-1) that are involved in lipid and glucose metabolism, but also results in the inhibition of genes encoding inflammatory factors such as tumor necrosis factor (TNF)- $\alpha$, interleukin (IL) $-1 \beta$, and interferon (IFN) $-\gamma^{1,11-15)}$

In the last decade, GlaxoSmithKline and other several pharmaceutical companies have been active in developing synthetic LXR agonists (Fig. 1). T0901317 (1, Tularik) and GW3965 (2, GSK) exhibit nonselectivity for LXR $\alpha$ and $\operatorname{LXR} \beta$ with high affinity. ${ }^{11,16-18)}$ The first compound to enter the clinic was LXR-623 (3, Wyeth), an LXR $\alpha / \beta$ partial agonist for the potential treatment of atherosclerosis and dyslipidemia. Unfortunately, the trial was terminated due to adverse central nervous system effects. ${ }^{19-21)}$ LXR antagonists reported so far include riccardin $\mathrm{C}(\mathbf{4}$, antagonist of $\mathrm{LXR} \beta)$, naringenin (5, antagonist of $\operatorname{LXR} \alpha$ ), genistein $(6$, inhibition of $\operatorname{LXR} \alpha$ or activation of $\operatorname{LXR} \beta)$, taurine (7, antagonist of $\operatorname{LXR} \alpha)$, rhein $(8$, antagonist of $\mathrm{LXR} \alpha / \beta)$, SR-9238 (9, antagonist of $\mathrm{LXR} \alpha / \beta)$, and 10 (antagonist of $\operatorname{LXR} \alpha$ ), among others ${ }^{22-28)}$ (Fig. 1).
SR-9238 was the first selective synthetic LXR inverse agonist that displays a degree of $\mathrm{LXR} \beta$ selectivity with an $\mathrm{IC}_{50}$ value of $214 \mathrm{~nm}$ for $\mathrm{LXR} \alpha$ and $43 \mathrm{~nm}$ for $\operatorname{LXR} \beta$, and this compound effectively suppressed hepatic lipogenesis, inflammation, and hepatic lipid accumulation in a mouse model of nonalcoholic hepatosteatosis. ${ }^{27)}$

In this paper, we describe the discovery and further structural development of LXR antagonists based on the fibrate skeleton. Both molecules activated LXRs in a luciferase reporter gene assay (GAL4) tested in HEK-293 cells. ${ }^{29)}$ In our previous studies, we first found that the combination of gemfibrozil and 4'-hydroxyacetophenone via the amide bond provided compound 15a (Fig. 2), which exhibited weak antagonistic activity toward $\operatorname{LXR} \alpha$ and $\operatorname{LXR} \beta$, and then we took ciprofibrate, bezafibrate, and fenofibrate as templates to design $\mathbf{1 5} \mathbf{b}-\mathbf{d}$ in the same manner as described for the preparation of 15a. Finally, the fenofibrate template was combined with substitued acetophenone to obtain further compounds as LXR antagonists.

\section{Results and Discussion}

Chemistry Compounds $\mathbf{1 5 a}-\mathbf{d}$ were synthesized starting with the preparation of 2-amino-1-(4-hydroxyphenyl)ethanone hydrochloride 13 by Delépine reaction at low temperature, followed by condensation of carboxylic acids $\mathbf{1 4 a}-\mathbf{d}$ in the presence of 1-(3-dimethylaminopropyl)-3-ethylcarbodiimide hydrochloride (EDCI $\cdot \mathrm{HCl})$ and 1-hydroxybenzotriazole (HOBT) at room temperature ${ }^{30,31)}$ (Chart 1). 14a and $\mathbf{d}$ were converted into 16a and $\mathbf{d}$ in the presence of $N, N^{\prime}$-carbonyldiimidazole (CDI) under an $\mathrm{H}_{2} \mathrm{~S}$ atmosphere, and then 14a, d, 16a, and $\mathbf{d}$ were allowed to react with 2-bromo-4'-hydroxyacetophenone 11 in the presence of $\mathrm{K}_{2} \mathrm{CO}_{3}{ }^{32,33)}$ (Chart 2). Compounds 22e-i were synthesized as shown in Chart 3, using a route nearly identical to that in Chart $1 .^{30,31)}$ The amide 24 was prepared by condensation of carboxylic acid $\mathbf{1 4 d}$ and 1-(tertbutoxycarbonyl)piperazine 23 with $\mathrm{EDCI} \cdot \mathrm{HCl}$ as a condensation agent and 4-dimethylaminopyridine (DMAP) as a catalyst in $\mathrm{CH}_{2} \mathrm{Cl}_{2} \cdot{ }^{34)}$ The deprotection of the tert-butoxycarbonyl (Boc) group on the amino group by hydrolysis with trifluoroacetic acid (TFA) was carried out at room temperature, then 
<smiles>CCCCC(C)(C)c1ccc(-c2c3cccc(C(F)(F)F)c3nn2Cc2ccc(F)cc2Cl)c(C(F)(F)F)c1</smiles><smiles>[R]c1ccc(CCc2ccc(Oc3cc(CCc4ccc(-c5ccc([R])cc5[R])c([R])c4)ccc3[R])cc2)cc1</smiles>

(4) Riccardin C: $\mathrm{R} 1=\mathrm{OH}, \mathrm{R} 2=\mathrm{OH}$<smiles>NCCS(=O)(=O)O</smiles>

(7) Taurine<smiles>O=C1C[C@H](c2ccc(O)cc2)Oc2cc(O)cc(O)c21</smiles>

(5) Naringenin<smiles>O</smiles><smiles>Cc1cc(O)c2c(=O)c(-c3ccc(O)cc3)coc2c1</smiles>

(6) Genistein<smiles>O=C(O)c1cc(O)c2c(c1)C(=O)c1cccc(O)c1C2=O</smiles>

(8) Rhein<smiles>Cn1c(=O)c2ccccc2c2cc(C(O)(C(F)(F)F)C(F)(F)F)ccc21</smiles>

(10)

Fig. 1. Chemical Structures of LXR Agonists (1-3) and LXR Antagonists (4-10)<smiles>C=CCCCCCC(C)(C)C(=O)O</smiles>

4'-hydroxyacetophenone<smiles>Cc1ccc(C)c(OCCCC(C)(C)C(=O)NCC(=O)c2ccc(O)cc2)c1</smiles>

15a<smiles>CCOC(=O)c1ccc(CN(C)S(=O)(=O)c2c(C)cc(C)cc2C)o1</smiles> 


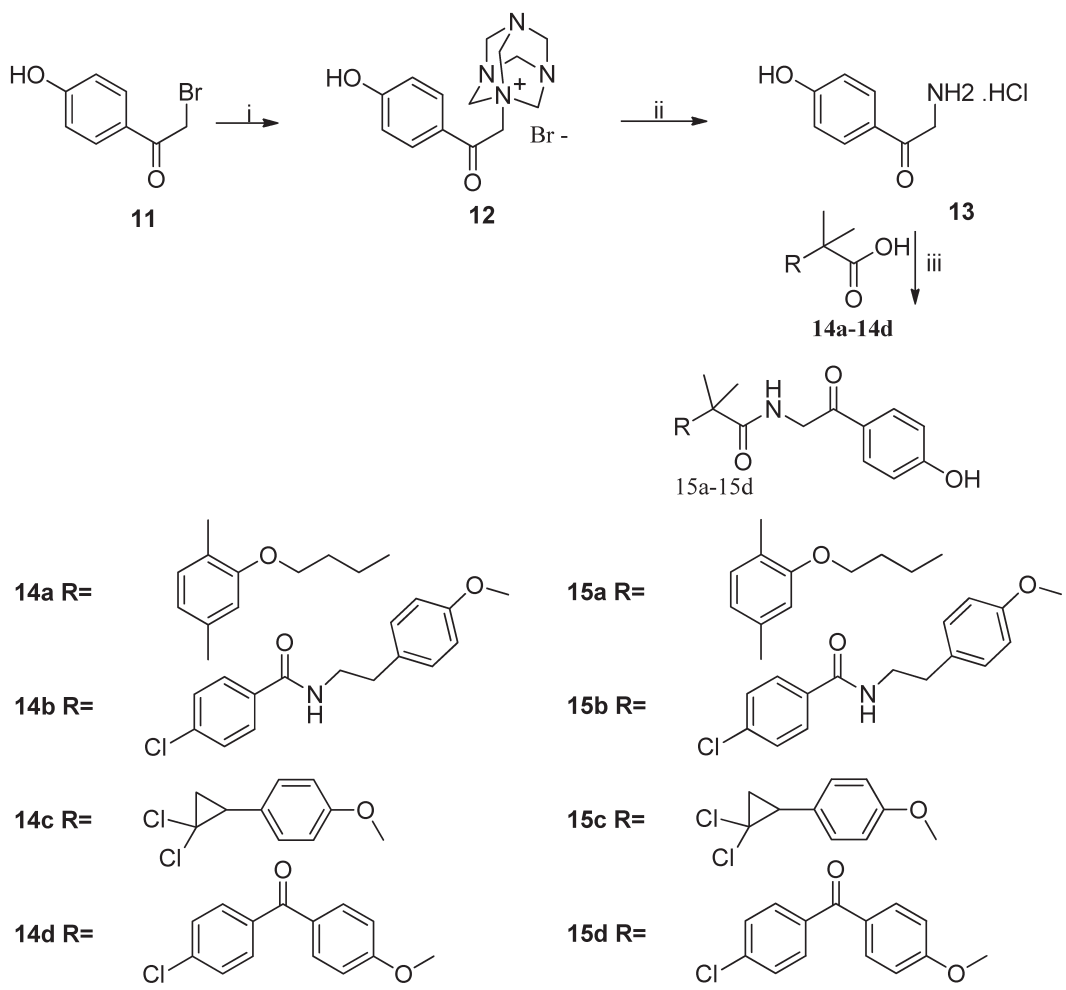

Reagents and conditions: (i) Hexamethylenetetramine, THF, rt; (ii) Conc. $\mathrm{HCl}$, ethanol, $45^{\circ} \mathrm{C}$; (iii) HOBT, EDCI $\cdot \mathrm{HCl}$, triethylamine, $\mathrm{CH}_{2} \mathrm{Cl}_{2}, \mathrm{rt}$. Chart 1. General Route for the Synthesis of $\mathbf{1 5 a}-\mathbf{d}$

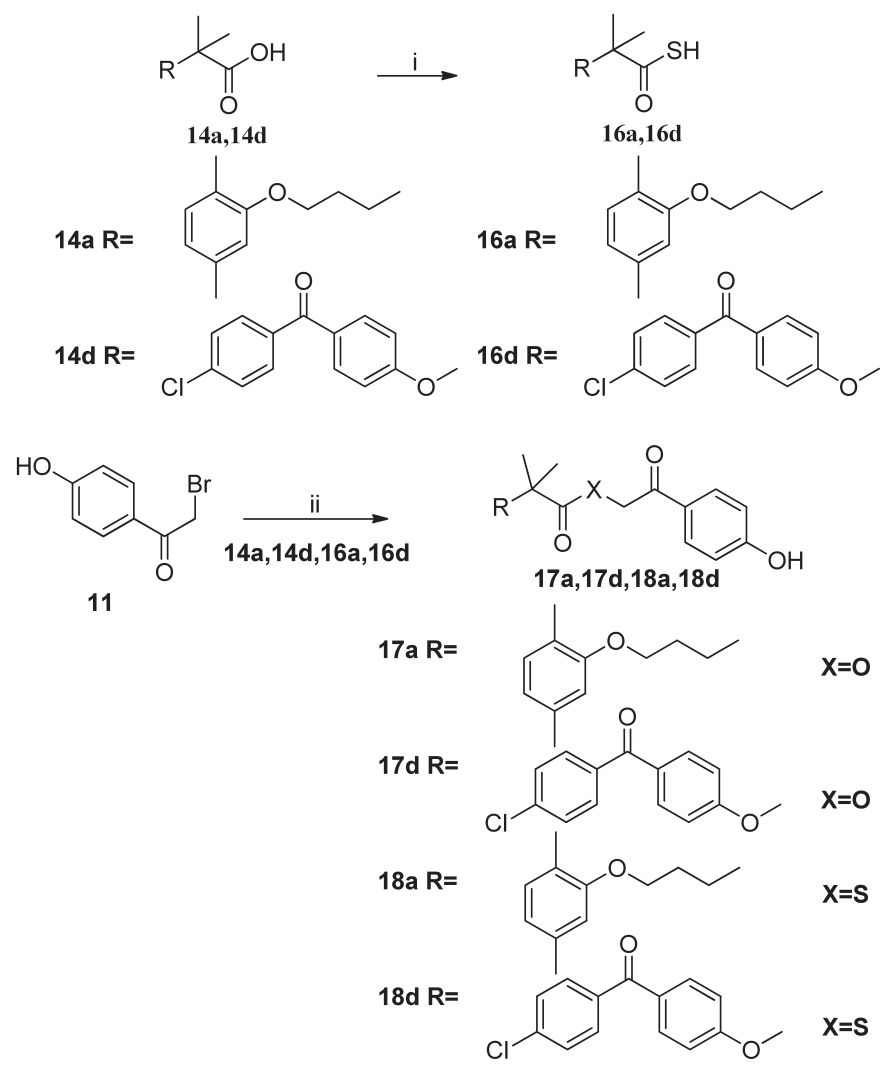

Reagents and conditions: (i) $\mathrm{CDI}, \mathrm{H}_{2} \mathrm{~S}$, DMF; (ii) $\mathrm{K}_{2} \mathrm{CO}_{3}$, THF.

Chart 2. General Route for the Synthesis of 17a, d, 18a, and $\mathbf{d}$ 
<smiles>[R][R]1cccc(C(=O)C[N+]23CN4CN(CN(C4)C2)C3)c1</smiles>

20

20e $m=4 R=F$

20f $m=4 R=H$

$20 \mathrm{~g} \mathrm{~m}=4 \mathrm{R}=\mathrm{CH} 3$

$20 \mathrm{~h} m=4 \mathrm{R}=\mathrm{SCH} 3$

20i $m=3 \mathrm{R}=\mathrm{OH}$

i<smiles></smiles>

19

19e $m=4 R=F$

$19 f m=4 R=H$

$19 \mathrm{~g} \mathrm{~m}=4 \mathrm{R}=\mathrm{CH} 3$

$19 \mathrm{~h} m=4 \mathrm{R}=\mathrm{SCH} 3$

19i $m=3 \mathrm{R}=\mathrm{OH}$

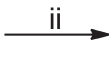

$\prod_{\mathrm{O}}^{\mathrm{N}} \mathrm{T}_{\mathrm{m}}^{\mathrm{NH}}$

21

21e $m=4 R=F$

$21 f m=4 R=H$

$21 \mathrm{~g} \mathrm{~m}=4 \mathrm{R}=\mathrm{CH} 3$

$21 \mathrm{~h} \mathrm{~m}=4 \mathrm{R}=\mathrm{SCH} 3$

21i $m=3 \mathrm{R}=\mathrm{OH}$

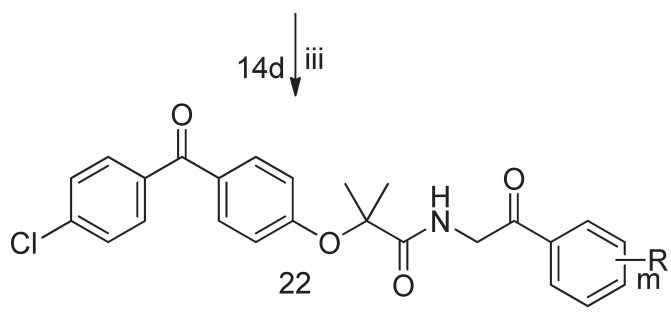

22e $m=4 R=F$

$22 f m=4 R=H$

22g $m=4 \mathrm{R}=\mathrm{CH} 3$

$22 \mathrm{~h} \mathrm{~m}=4 \mathrm{R}=\mathrm{SCH} 3$

22i $m=3 \mathrm{R}=\mathrm{OH}$

Reagents and conditions: (i) Hexamethylenetetramine, THF, rt; (ii) Conc. $\mathrm{HCl}$, ethanol, $45^{\circ} \mathrm{C}$; (iii) $\mathrm{HOBT}$, $\mathrm{EDCI} \cdot \mathrm{HCl}$, triethylamine, $\mathrm{CH}_{2} \mathrm{Cl}_{2}, \mathrm{rt}$.

Chart 3. General Route for the Synthesis of $\mathbf{2 2} \mathbf{e}-\mathbf{i}$<smiles>CC(C)(C)OC(=O)N1CCNCC1</smiles><smiles>[Z20]c1ccc(C(=O)c2ccc(OC(C)(C)C(=O)N3CCN(C(=O)OC(C)(C)C)CC3)cc2)cc1</smiles>

Reagents and conditions: (i) EDCI·HCl, DMAP, triethylamine, $\mathrm{CH}_{2} \mathrm{Cl}_{2}$; (ii) TFA, THF; (iii) $\mathrm{K}_{2} \mathrm{CO}_{3}$, THF.

Chart 4. General Route for the Synthesis of Compound 26

Table 1. Inhibition (\%) by $\mathbf{1 5 a}-\mathbf{d}$ at $25 \mu \mathrm{M}$ of $\mathrm{LXRs}^{a}{ }^{a}$

\begin{tabular}{crr}
\hline \hline Compound & LXR $\alpha$ & \multicolumn{1}{c}{$\operatorname{LXR} \beta$} \\
\hline $\mathbf{1 5 a}$ & $35.6 \pm 2.7$ & $26.8 \pm 6.4$ \\
$\mathbf{1 5 b}$ & $12.9 \pm 1.8$ & $8.4 \pm 4.9$ \\
$\mathbf{1 5 c}$ & $4.4 \pm 4.1$ & $-13.6 \pm 1.4$ \\
$\mathbf{1 5 d}$ & $57.6 \pm 2.0$ & $61.2 \pm 5.1$
\end{tabular}

a) Results are given as the mean \pm S.D. of two independent experiments.

might possess potent antagonistic activity against LXRs. Therefore another six compounds were prepared and their antagonistic activities were determined in vitro (Table 3), using clotrimazole ${ }^{37)}$ as the standard reference. With a longer chain, compound 26 had an $\mathrm{IC}_{50}$ value of $6.4 \mu \mathrm{M}$ for $\mathrm{LXR} \alpha$ and $5.6 \mu \mathrm{M}$ for $\operatorname{LXR} \beta$, which was two-fold more potent than 15d, while compound $22 \mathrm{~g}$ had an $\mathrm{IC}_{50}$ value of $30.6 \mu \mathrm{M}$ for LXR $\alpha$ and 19.2 $\mu \mathrm{M}$ for LXR $\beta$. Compounds $22 \mathbf{e}, \mathbf{f}, \mathbf{h}$, and $\mathbf{i}$ also showed good antagonistic activity, although not as effective as 15d. The structure-activity relationship (SAR) indicating the extension of the amide linker for this class may enhance the
Table 2. Inhibition (\%) by 15a, d, 17a, d, 18a, d, Gemfibrozil, and Fenofibrate at $25 \mu \mathrm{M}$ of $\mathrm{LXRs}^{a}$

\begin{tabular}{ccc}
\hline \hline Compound & LXR $\alpha$ & LXR $\beta$ \\
\hline $\mathbf{1 5 a}$ & $38.9 \pm 3.0$ & $30.2 \pm 4.2$ \\
$\mathbf{1 7 a}$ & $18.8 \pm 1.9$ & $23.6 \pm 12.2$ \\
$\mathbf{1 8 a}$ & $0.8 \pm 4.5$ & $7.6 \pm 5.8$ \\
Gemfibrozil & $-4.3 \pm 9.7$ & $-10.4 \pm 10.6$ \\
$\mathbf{1 5 d}$ & $68.0 \pm 14.0$ & $65.2 \pm 6.7$ \\
$\mathbf{1 7 d}$ & $38.4 \pm 5.2$ & $43.4 \pm 3.1$ \\
$\mathbf{1 8 d}$ & $17.5 \pm 5.7$ & $24.3 \pm 7.0$ \\
Fenofibrate & $-20.7 \pm 8.7$ & $-14.8 \pm 5.5$
\end{tabular}

a) Results are given as the mean \pm S.D. of two independent experiments.

antagonistic activity against LXRs.

Molecular Docking The predicted interaction between the protein and ligand is shown in Fig. 3. For $\operatorname{LXR} \alpha$, the phenolic hydroxyl group formed a strong hydrogen bond with His421, and the substituted phenolic hydroxyl group is seen as a hydrogen bond to Thr302. The left-hand side phenolic 
hydroxyl phenyl ring is embedded in a hydrophobic pocket formed by Leu331, Phe335, and Leu428, while the righthand side chlorine-substituted phenyl ring is inserted into a hydrophobic pocket formed by Leu260, Ser264, Phe315, and Leu316. Also, a pair of $\pi-\pi$ interactions exists between the middle phenyl ring and Phe315. For $\operatorname{LXR} \beta$, the phenolic hydroxyl group formed a strong hydrogen bond with Ala343, while the left-hand side phenolic hydroxyl phenyl ring occupied a hydrophobic pocket formed by Phe268, Leu345, and Leu442, and the right-hand side conjugated phenyl ring was embedded in a hydrophobic pocket formed by Ser278, Met312, Thr316, Phe329, and Leu330.

\section{Conclusion}

In summary, we synthesized 14 novel compounds that exhibited different antagonistic activitives against $\mathrm{LXR} \alpha / \beta$. Among these compounds, compound $\mathbf{2 6}$ had antagonistic activity two-fold more potent than $\mathbf{1 5 d}$ toward LXR $\alpha / \beta$ and its binding was predicted. SAR studies indicated that compounds with the amide linker were more potent than compounds with ester and thioester linkers. Furthermore, a longer chain between the fenofibrate template and the acetophenone template may enhance the antagonistic activity. Further research is required to optimize this scaffold for the design of more potent $\mathrm{LXR} \alpha / \beta$ antagonists.

\section{Experimental}

Chemistry Melting points were determined on a WRS-21 melting point apparatus (Shanghai Shen Guang Instrument Co., Ltd., Shanghai, P.R. China) and were uncorrected.

Table 3. $\mathrm{IC}_{50}$ Values $(\mu \mathrm{M})$ of $\mathbf{1 5 d}, \mathbf{2 2} \mathbf{e}-\mathbf{i}$, and $\mathbf{2 6}$ against $\left.\mathrm{LXRs}^{a}\right)$

\begin{tabular}{ccc}
\hline \hline Compound & LXR $\alpha$ & LXR $\beta$ \\
\hline $\mathbf{1 5 d}$ & 12.2 & 12.7 \\
$\mathbf{2 2} \mathbf{e}$ & 16.9 & 14.8 \\
$\mathbf{2 2 f}$ & 15.9 & 16.2 \\
$\mathbf{2 2} \mathbf{g}$ & 30.6 & 19.2 \\
$\mathbf{2 2} \mathbf{h}$ & 16.1 & 15.2 \\
$\mathbf{2 2}$ & 13.2 & 17.6 \\
$\mathbf{2 6}$ & 6.4 & 5.6 \\
Clotrimazole $^{b)}$ & 10.1 & 11.8
\end{tabular}

a) Results are given as the mean of two independent experiments. b) Standard reference.

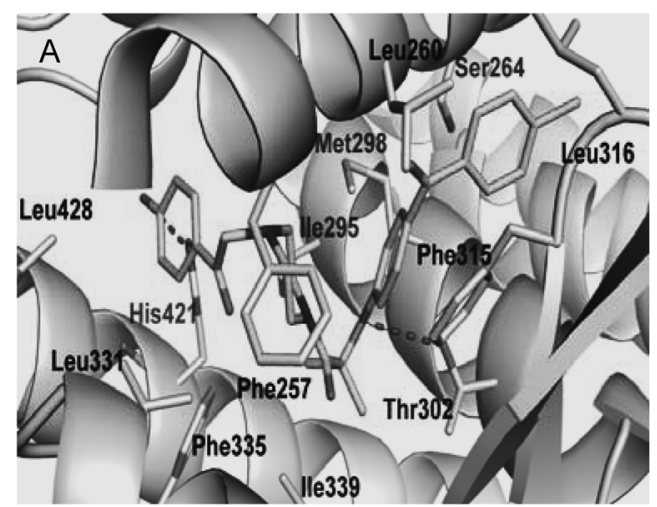

${ }^{1} \mathrm{H}-\mathrm{NMR}$ spectra were recorded on an INOVA $400(400-\mathrm{MHz})$ spectrometer (Varian Inc., Palo Alto, CA, U.S.A.) with tetramethylsilane (TMS) as an internal standard. Chemical shifts $(\delta)$ are in ppm relative to TMS, and coupling constants $(J)$ are expressed in hertz $(\mathrm{Hz})$. Electron-spray ionization mass spectra (ESI-MS) in positive mode were recorded on a HP5989A mass spectrometer (Hewlett-Packard, Palo Alto, CA, U.S.A.). The purity of all novel compounds was checked using TLC and ${ }^{1} \mathrm{H}-\mathrm{NMR}$. All reactions were monitored using TLC on precoated Silica Gel F254 plates (Yantai Jiang You Silicone Development Co., Ltd., Yantai, P. R. China) with detection by UV. All reagents used were of analytical grade (J\&K Scientific Ltd., Beijing, P. R. China, Aladdin Industrial Inc., Shanghai, P. R. China, or Sinopharm Chemical Reagent Co., Ltd., Beijing, P. R. China).

General Procedure for the Synthesis of $\mathbf{1 5 a}-\mathbf{d}^{30,31)}$ To a solution of 2-bromo-4'-hydroxyacetophenone $\mathbf{1 1}(20.0 \mathrm{mmol})$ in tetrahydrofuran (THF) $(50 \mathrm{~mL})$, hexamethylenetetramine $(20.0 \mathrm{mmol})$ was added and stirred for $3 \mathrm{~h}$ at room temperature, and then the precipitated hexamethylenetetramine adduct 12 was filtered out. The adduct $\mathbf{1 2}$ was then heated with ethanol $(80 \mathrm{~mL})$ and concentrated $\mathrm{HCl}(8 \mathrm{~mL})$ for $1 \mathrm{~h}$ at $45^{\circ} \mathrm{C}$. After cooling, the inorganics were filtered out, the mixture was washed with ethanol $(20 \mathrm{~mL})$, and the solvent was distilled out completely under reduced pressure to obtain the desired compound 13. Then $14 \mathbf{a}-\mathbf{d}(2.0 \mathrm{mmol})$, triethylamine $(4.0 \mathrm{mmol})$, and $\mathrm{EDCI} \cdot \mathrm{HCl}(4.0 \mathrm{mmol})$, followed by $\mathrm{HOBT}(4.0 \mathrm{mmol})$, were added to a stirred solution of $\mathbf{1 3}(2.0 \mathrm{mmol})$ in $\mathrm{CH}_{2} \mathrm{Cl}_{2}$ $(10 \mathrm{~mL})$ and the mixture was stirred for $12 \mathrm{~h}$ at room temperature. Saturated $\mathrm{Na}_{2} \mathrm{CO}_{3}$ was added, the mixture was extracted with ethyl acetate, and the extracts were washed with brine, dried over $\mathrm{MgSO}_{4}$, filtered, and concentrated. The residue was purified by chromatography to give target compounds $\mathbf{1 5 a}-\mathbf{d}$.

5-(2,5-Dimethylphenoxy)- $N$-(2-(4-hydroxyphenyl)-2-oxoethyl)2,2-dimethylpentanamide (15a): Yield 75\%, mp $131.2-134.3^{\circ} \mathrm{C}$. ${ }^{1} \mathrm{H}-\mathrm{NMR}\left(400 \mathrm{MHz}, \mathrm{CDCl}_{3}\right) \delta: 1.16(6 \mathrm{H}, \mathrm{s}), 1.62-1.73(4 \mathrm{H}, \mathrm{m})$, $2.10(3 \mathrm{H}, \mathrm{s}), 2.25(3 \mathrm{H}, \mathrm{s}), 3.89-3.92(2 \mathrm{H}, \mathrm{m}), 4.44-4.46(2 \mathrm{H}, \mathrm{d}$, $J=5.6 \mathrm{~Hz}), 6.61-6.63(1 \mathrm{H}, \mathrm{d}, J=8.0 \mathrm{~Hz}), 6.71(1 \mathrm{H}, \mathrm{s}), 6.85-6.99$ $(2 \mathrm{H}, \mathrm{m}), 7.67-7.69(1 \mathrm{H}, \mathrm{m}), 7.85-7.88(2 \mathrm{H}, \mathrm{m}), 10.30(1 \mathrm{H}, \mathrm{s})$. ESI-MS $m / z: 384[\mathrm{M}+\mathrm{H}]^{+}$.

4-Chloro- $N$-(4-((1-((2-(4-hydroxyphenyl)-2-oxoethyl)amino)2-methyl-1-oxopropan-2-yl)oxy)phenethyl)benzamide (15b): Yield $74 \%$, mp 216.0-220.4 ${ }^{\circ} \mathrm{C}$. ${ }^{1} \mathrm{H}-\mathrm{NMR}\left(400 \mathrm{MHz}, \mathrm{CDCl}_{3}\right) \delta: 1.38$ $(6 \mathrm{H}, \mathrm{s}), 2.73-2.76(2 \mathrm{H}, \mathrm{m}), 3.47-3.48(2 \mathrm{H}, \mathrm{m}), 4.55-4.56(2 \mathrm{H}$,

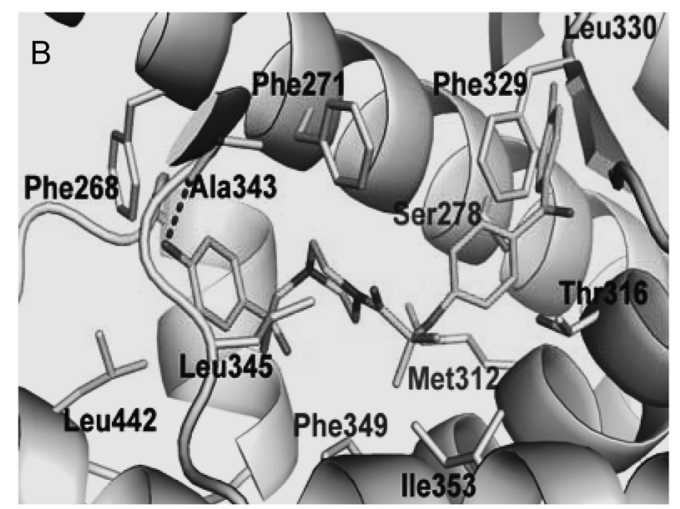

Fig. 3. Binding Modes of $\mathbf{2 6}$ to $\operatorname{LXR} \alpha$ and $\operatorname{LXR} \beta$

(A) Binding mode of $\mathbf{2 6}$ within the LXR $\alpha$-LBD; (B) Binding mode of $\mathbf{2 6}$ within the LXR $\beta$-LBD. The ligand is depicted as sticks, amino acids involved in ligand binding are shown as gray sticks, and hydrogen bonds are denoted by dotted lines. 
$\mathrm{d}, J=4.8 \mathrm{~Hz}), 6.75-6.81(4 \mathrm{H}, \mathrm{m}), 6.99-7.01(2 \mathrm{H}, \mathrm{d}, J=8.0 \mathrm{~Hz})$, $7.16(1 \mathrm{H}, \mathrm{s}), 7.21-7.23(2 \mathrm{H}, \mathrm{d}, J=8.8 \mathrm{~Hz}), 7.21(1 \mathrm{H}, \mathrm{s}), 7.58-7.61$ $(2 \mathrm{H}, \mathrm{d}, J=8.4 \mathrm{~Hz}), 7.63(1 \mathrm{H}, \mathrm{s}), 7.70-7.72(2 \mathrm{H}, \mathrm{d}, J=8.8 \mathrm{~Hz})$. ESI-MS $m / z: 495[\mathrm{M}+\mathrm{H}]^{+}$.

2-(4-(2,2-Dichlorocyclopropyl)phenoxy)- $N$-(2-(4-hydroxyphenyl)-2-oxoethyl)-2-methylpropanamide (15c): Yield 72\%, mp 113.6-115.9 ${ }^{\circ}$ C. ${ }^{1} \mathrm{H}-\mathrm{NMR}\left(400 \mathrm{MHz}, \mathrm{CDCl}_{3}\right) \delta: 1.55(6 \mathrm{H}$, s), 1.93-1.97 (2H, m), 2.82-2.88 $(1 \mathrm{H}, \mathrm{m}), 4.71-4.72(2 \mathrm{H}, \mathrm{d}$, $J=4.8 \mathrm{~Hz}), 6.89-6.99$ (4H, m), 7.15-7.16 (2H, d, $J=5.2 \mathrm{~Hz})$, $7.72(1 \mathrm{H}, \mathrm{s}), 7.88-7.90(2 \mathrm{H}, \mathrm{d}, J=8.8 \mathrm{~Hz})$. ESI-MS $\mathrm{m} / \mathrm{z}: 422$ $[\mathrm{M}+\mathrm{H}]^{+}$.

2-(4-(4-Chlorobenzoyl)phenoxy)- $N$-(2-(4-hydroxyphenyl)-2oxoethyl)-2-methylpropanamide (15d): Yield 65\%, mp 172.4-174. $3^{\circ} \mathrm{C} .{ }^{1} \mathrm{H}-\mathrm{NMR}\left(400 \mathrm{MHz}, \mathrm{CDCl}_{3}\right) \delta$ : $1.67(6 \mathrm{H}, \mathrm{s})$, $4.70-4.71(2 \mathrm{H}, \mathrm{d}, J=4.8 \mathrm{~Hz}), 6.64(1 \mathrm{H}, \mathrm{s}), 6.91-6.93(2 \mathrm{H}, \mathrm{d}$, $J=8.8 \mathrm{~Hz}), 7.05-7.07(2 \mathrm{H}, \mathrm{m}), 7.45-7.47(2 \mathrm{H}, \mathrm{d}, J=8.4 \mathrm{~Hz}), 7.61$ $(1 \mathrm{H}, \mathrm{s}), 7.71-7.83(6 \mathrm{H}, \mathrm{m})$. ESI-MS $m / z: 452[\mathrm{M}+\mathrm{H}]^{+}$.

General Procedure for the Synthesis of 17a, d, 18a, and $\mathbf{d}^{32,33)}$ CDI $(10.0 \mathrm{mmol})$ was dissolved in $N, N$-dimethylformamide (DMF) $(20 \mathrm{~mL})$ and to this solution a solution of the carboxylic acids $\mathbf{1 4 a}$ and $\mathbf{d}(10.0 \mathrm{mmol})$ in DMF $(2 \mathrm{~mL})$ was added dropwise. Subsequently, the reaction mixture was stirred at room temperature for $1 \mathrm{~h}$. Then, $\mathrm{H}_{2} \mathrm{~S}$ was bubbled gently through the reaction mixture for $2 \mathrm{~h}$. Sulfuric acid $(0.5 \mathrm{M}, 40 \mathrm{~mL})$ was added and the mixture was extracted with ethyl acetate. The organic layer was dried with magnesium sulfate $\left(\mathrm{MgSO}_{4}\right)$, filtered, and concentrated to yield 16a, d, 14a, d, 16a, and $\mathbf{d}(2.0 \mathrm{mmol})$. They were dissolved in THF $(10 \mathrm{~mL})$, anhydrous $\mathrm{K}_{2} \mathrm{CO}_{3}(4.0 \mathrm{mmol})$ and 2-bromo-4'hydroxyacetophenone $\mathbf{1 1}(2.0 \mathrm{mmol})$ were added, and the mixture was stirred for $4 \mathrm{~h}$ at room temperature. Dilute $\mathrm{HCl}$ was added, and the mixture was extracted with ethyl acetate, washed with brine, dried over $\mathrm{MgSO}_{4}$, filtered, and concentrated, and the residue was purified by chromatography to afford target compounds $17 \mathbf{a}, \mathbf{d}, \mathbf{1 8 a}$, and $\mathbf{d}$.

2-(4-Hydroxyphenyl)-2-oxoethyl-5-(2,5-dimethylphenoxy)2,2-dimethylpentanoate (17a): Yield $83 \%$, mp $105.6-108.3^{\circ} \mathrm{C}$. ${ }^{1} \mathrm{H}-\mathrm{NMR}\left(400 \mathrm{MHz}, \mathrm{CDCl}_{3}\right) \delta: 1.32(6 \mathrm{H}, \mathrm{s}), 1.81-1.85(4 \mathrm{H}, \mathrm{m})$, $2.18(3 \mathrm{H}, \mathrm{s}), 2.30(3 \mathrm{H}, \mathrm{s}), 3.94-3.97(2 \mathrm{H}, \mathrm{m}), 5.23(2 \mathrm{H}, \mathrm{s}), 5.62$ $(1 \mathrm{H}, \mathrm{s}), 6.63-6.66(2 \mathrm{H}, \mathrm{m}), 6.85-6.87(2 \mathrm{H}, \mathrm{m}), 6.98-7.00(1 \mathrm{H}$, d, $J=7.6 \mathrm{~Hz}), 7.80-7.82(2 \mathrm{H}, \mathrm{m})$. ESI-MS $m / z: 385[\mathrm{M}+\mathrm{H}]^{+}$.

2-(4-Hydroxyphenyl)-2-oxoethyl-2-(4-(4-chlorobenzoyl)phenoxy)-2-methylpropanoate (17d): Yield $89 \%, \quad \mathrm{mp}$ 112.0-115.2 ${ }^{\circ} \mathrm{C} .{ }^{1} \mathrm{H}-\mathrm{NMR}\left(400 \mathrm{MHz}, \mathrm{CDCl}_{3}\right) \delta: 1.79(6 \mathrm{H}, \mathrm{s})$, $5.35(2 \mathrm{H}, \mathrm{s}), 6.21(1 \mathrm{H}, \mathrm{s}), 6.87-6.88(2 \mathrm{H}, \mathrm{m}), 7.02-7.26(2 \mathrm{H}$, m), 7.44-7.46 (2H, d, $J=8.8 \mathrm{~Hz}), 7.70-7.81(6 \mathrm{H}, \mathrm{m})$. ESI-MS $m / z: 453[\mathrm{M}+\mathrm{H}]^{+}$.

$S$-(2-(4-Hydroxyphenyl)-2-oxoethyl)-5-(2,5-dimethylphenoxy)-2,2-dimethylpentanethioate (18a): Yield 67\%, mp 90.3-93.9 ${ }^{\circ} \mathrm{C} .{ }^{1} \mathrm{H}-\mathrm{NMR}\left(400 \mathrm{MHz}, \mathrm{CDCl}_{3}\right) \delta: 1.32(6 \mathrm{H}, \mathrm{s})$, 1.84-1.86 (4H, m), $2.18(3 \mathrm{H}, \mathrm{s}), 2.30(3 \mathrm{H}, \mathrm{s}), 3.94-3.97(2 \mathrm{H}$, m), $5.24(2 \mathrm{H}, \mathrm{s}), 5.68(1 \mathrm{H}, \mathrm{s}), 6.63-6.66(2 \mathrm{H}, \mathrm{m}), 6.86-6.88$ (2H, m), 6.99-7.01 (1H, d, J=7.2 Hz), 7.80-7.82 (2H, m). ESIMS $m / z: 423[\mathrm{M}+\mathrm{Na}]^{+}$.

$S$-(2-(4-Hydroxyphenyl)-2-oxoethyl)-2-(4-(4-chlorobenzoyl)phenoxy)-2-methylpropanethioate (18d): Yield 57\%, mp 150.1-153.6 ${ }^{\circ} \mathrm{C} .{ }^{1} \mathrm{H}-\mathrm{NMR} \quad\left(400 \mathrm{MHz}, \mathrm{CDCl}_{3}\right) \delta: 1.65(6 \mathrm{H}$, s), $4.33(2 \mathrm{H}, \mathrm{s}), 5.57(1 \mathrm{H}, \mathrm{s}), 6.88-6.90(2 \mathrm{H}, \mathrm{d}, J=8.8 \mathrm{~Hz})$, 6.99-7.01 (2H, d, $J=8.8 \mathrm{~Hz}), 7.45-7.47(2 \mathrm{H}, \mathrm{d}, J=8.4 \mathrm{~Hz})$, 7.71-7.74 (2H, m), 7.91-7.93 (2H, d,=8.4 Hz). ESI-MS m/z: 491 $[\mathrm{M}+\mathrm{Na}]^{+}$.
General Procedure for the Synthesis of $22 \mathrm{e}-\mathbf{i}^{30,31)}$ Compounds $22 \mathbf{e}-\mathbf{i}$ were prepared from $19 \mathbf{e}-\mathbf{i}$ by means of a procedure similar to that used for $\mathbf{1 5 a}-\mathbf{d}$.

2-(4-(4-Chlorobenzoyl)phenoxy)- $N$-(2-(4-fluorophenyl)-2oxoethyl)-2-methylpropanamide (22e): Yield 82\%, mp 125.8-125.9 ${ }^{\circ}$. ${ }^{1} \mathrm{H}-\mathrm{NMR}\left(400 \mathrm{MHz}, \mathrm{CDCl}_{3}\right) \delta: 1.66(6 \mathrm{H}, \mathrm{s})$, $4.75-4.78(2 \mathrm{H}, \mathrm{d}, J=5.2 \mathrm{~Hz}), 7.04-7.06(2 \mathrm{H}, \mathrm{d}, J=8.8 \mathrm{~Hz})$, $7.15-7.20(2 \mathrm{H}, \mathrm{m}), 7.44-7.48(2 \mathrm{H}, \mathrm{m}), 7.70-7.77$ (4H, m), 7.99-8.02 (2H, m). ESI-MS m/z: $476[\mathrm{M}+\mathrm{Na}]^{+}$.

2-(4-(4-Chlorobenzoyl)phenoxy)-2-methyl- $\mathrm{N}$-(2-oxo-2phenylethyl)propanamide (22f): Yield $89 \%$, mp $141.2-143.3^{\circ} \mathrm{C}$. ${ }^{1} \mathrm{H}-\mathrm{NMR}\left(400 \mathrm{MHz}, \mathrm{CDCl}_{3}\right) \delta: 1.66(6 \mathrm{H}, \mathrm{s}), 4.79-4.80(2 \mathrm{H}$, $\mathrm{d}, J=4.8 \mathrm{~Hz}), 7.04-7.07(2 \mathrm{H}, \mathrm{m}), 7.44-7.52(5 \mathrm{H}, \mathrm{m}), 7.61-7.64$ $(1 \mathrm{H}, \mathrm{m}), 7.71-7.77$ (4H, m), 7.96-7.98 (2H, m). ESI-MS $\mathrm{m} / \mathrm{z}$ : $436[\mathrm{M}+\mathrm{H}]^{+}$.

2-(4-(4-Chlorobenzoyl)phenoxy)-2-methyl- $N$-(2-oxo-2-( $p$ tolyl)ethyl)propanamide (22g): Yield 83\%, mp $124.0-124.3^{\circ} \mathrm{C}$. ${ }^{1} \mathrm{H}-\mathrm{NMR}\left(400 \mathrm{MHz}, \mathrm{CDCl}_{3}\right) \delta: 1.65(6 \mathrm{H}, \mathrm{s}), 2.42(3 \mathrm{H}, \mathrm{s})$, $4.75-4.76(2 \mathrm{H}, \mathrm{d}, J=4.4 \mathrm{~Hz}), 7.03-7.05(2 \mathrm{H}, \mathrm{d}, J=8.0 \mathrm{~Hz})$, $7.25-7.30(2 \mathrm{H}, \mathrm{m}), 7.43-7.45(2 \mathrm{H}, \mathrm{d}, J=8.4 \mathrm{~Hz}), 7.51(1 \mathrm{H}$, s), 7.70-7.76 (4H, m), 7.85-7.87 (2H, m). ESI-MS $m / z: 472$ $[\mathrm{M}+\mathrm{Na}]^{+}$.

2-(4-(4-Chlorobenzoyl)phenoxy)-2-methyl-N-(2-(4(methylthio)phenyl)-2-oxoethyl)propanamide (22h): Yield 89\%, mp 113.9-114. $6^{\circ} \mathrm{C} .{ }^{1} \mathrm{H}-\mathrm{NMR}\left(400 \mathrm{MHz}, \mathrm{CDCl}_{3}\right) \delta: 1.69(6 \mathrm{H}, \mathrm{s})$, $2.56(3 \mathrm{H}, \mathrm{s}), 4.77-4.78(2 \mathrm{H}, \mathrm{d}, J=4.4 \mathrm{~Hz}), 7.07-7.09(2 \mathrm{H}, \mathrm{d}$, $J=8.8 \mathrm{~Hz}), 7.32-7.34(2 \mathrm{H}, \mathrm{d}, J=8.0 \mathrm{~Hz}), 7.46-7.47(2 \mathrm{H}, \mathrm{m})$, $7.54(1 \mathrm{H}, \mathrm{s}), 7.74-7.80(4 \mathrm{H}, \mathrm{m}), 7.89-7.91(2 \mathrm{H}, \mathrm{d}, J=8.8 \mathrm{~Hz})$. ESI-MS $m / z: 504[\mathrm{M}+\mathrm{Na}]^{+}$.

2-(4-(4-Chlorobenzoyl)phenoxy)- $N$-(2-(3-hydroxyphenyl)-2oxoethyl)-2-methylpropanamide (22i): Yield 69\%, mp 168.1-172. $3^{\circ} \mathrm{C} .{ }^{1} \mathrm{H}-\mathrm{NMR} \quad\left(400 \mathrm{MHz}, \mathrm{CDCl}_{3}\right) \quad \delta: 1.53(6 \mathrm{H}$, s), $3.26(2 \mathrm{H}, \mathrm{s}), 7.00-7.01(4 \mathrm{H}, \mathrm{m}), 7.24(1 \mathrm{H}, \mathrm{s}), 7.51(1 \mathrm{H}$, s), 7.59-7.62 (4H, m), 7.70-7.73 (4H, m). ESI-MS $m / z: 474$ $[\mathrm{M}+\mathrm{Na}]^{+}$.

General Procedure for the Synthesis of $\mathbf{2 6}^{34-36)}$ DMAP $(2.0 \mathrm{mmol})$ and $\mathrm{EDCI} \cdot \mathrm{HCl}(40.0 \mathrm{mmol})$ were added to a solution of 1-(tert-butoxycarbonyl) piperazine $23(20.0 \mathrm{mmol})$ and carboxylic acid $\mathbf{1 4 d}(20.0 \mathrm{mmol})$ in $\mathrm{CH}_{2} \mathrm{Cl}_{2}(125 \mathrm{~mL})$. The reaction mixture was stirred at room temperature for $12 \mathrm{~h}$ and then washed with dilute $\mathrm{HCl}$ and water. The organic layer was dried over $\mathrm{MgSO}_{4}$, filtered, and the filtrate concentrated in vacuo to provide 24. Compound $24(2.0 \mathrm{mmol})$ was dissolved in THF $(10 \mathrm{~mL})$, TFA $(1 \mathrm{~mL})$ was added, and the resulting mixture shaken at room temperature for $24 \mathrm{~h}$. The reaction mixture was concentrated at reduced pressure and basified with $\mathrm{NaOH}$ solution (until $\mathrm{pH} 10$ ), extracted with $\mathrm{CH}_{2} \mathrm{Cl}_{2}$, dried over $\mathrm{MgSO}_{4}$, filtered, and evaporated at reduced pressure, and then the residue was purified by chromatography to afford 25. $\mathrm{K}_{2} \mathrm{CO}_{3}(4.0 \mathrm{mmol})$ and $\mathbf{1 1}(2.0 \mathrm{mmol})$ were added to a solution of $25(2.0 \mathrm{mmol})$ in THF $(10 \mathrm{~mL})$, and the reaction mixture was stirred at room temperature for $12 \mathrm{~h}$, filtered, and evaporated at reduced pressure. The residue was then purified by chromatography to afford target compound $\mathbf{2 6}$.

2-(4-(4-Chlorobenzoyl)phenoxy)-1-(4-(2-(4-hydroxyphenyl)-2-oxoethyl)piperazin-1-yl)-2-methylpropan-1-one (26): Yield $37 \%$, mp $225.6-228.3^{\circ} \mathrm{C}$. ${ }^{1} \mathrm{H}-\mathrm{NMR}$ (400 MHz, DMSO$\left.d_{6}\right) \delta: 1.61(6 \mathrm{H}, \mathrm{s}), 2.12(2 \mathrm{H}, \mathrm{s}), 2.39(2 \mathrm{H}, \mathrm{s}), 3.51-3.68(6 \mathrm{H}$, t), 6.78-6.80 (2H, d), 6.91-6.94 (2H, d), 7.58-7.61 (2H, d), 7.71-7.81 (6H, m), $10.36(1 \mathrm{H}, \mathrm{s})$. ESI-MS m/z: $521[\mathrm{M}+\mathrm{H}]^{+}$.

Biology Materials HEK293 cells were purchased from 
the ATCC (Manassas, VA, U.S.A.). Dulbecco's modified Eagle's medium (DMEM), fetal bovine serum (FBS), ethylenediaminetetraacetic acid (EDTA), phosphate buffered saline (PBS), and OPTI MEM I were purchased from Invitrogen (Carlsbad, CA, U.S.A.). TO901317 and clotrimazole were purchased from Sigma-Aldrich (St. Louis, MO, U.S.A.). pBind-LXR $\alpha$ and pBind-LXR $\beta$ and were constructed by Chempartner (Shanghai, P. R. China). Fugene HD Transfection Reagent, pG5Luc plasmid, and the Dual Luciferase Reporter Assay System were purchased from Promega (Madison, WI, U.S.A.).

LXR $\alpha(\beta) / p G 5 L u c$ Dual Luciferase Reporter Assay Prior to transfection, HEK293 cells were maintained in DMEM supplemented with 10\% FBS in regular tissue culture flasks. The host cells were plated in a 96-well tissue culture plate at a density of $5 \times 10^{-4}$ cells per well. The transfection mixture contained $25 \mathrm{ng}$ of $\mathrm{pBind}-\mathrm{LXR} \alpha$ (or $\mathrm{pBind}-\mathrm{LXR} \beta$ ) and $25 \mathrm{ng}$ of pG5Luc using $0.15 \mu \mathrm{L}$ of FuGENE HD transfection reagent per well. Twenty-four hours posttransfection, the transfection medium was removed before $1000 \mathrm{~nm}$ of TO901317 and various concentrations of test chemicals dissolved in medium were added for the measurement of antagonistic activity. After treatment with test chemicals for $24 \mathrm{~h}$, the cells were harvested and analyzed immediately using a 96-well plate (Shanghai Bioleaf Biotech Co., Ltd., Shanghai, P.R. China) luminometer. The amounts of firefly luciferase and renilla luciferase were measured with the Dual Luciferase Reporter Assay System kit (Progema) following the manufacturer's instructions. The value of luciferase for each lysate was normalized to the renilla luciferase activity. The percentage of inhibition was calculated using the following formula:

$\%$ Inhibition

$=\left[\frac{1000 \mathrm{~nm} \text { TO901317 (firefly/renilla) }- \text { chemical (firefly } / \text { renilla })}{1000 \mathrm{~nm} \text { TO901317 (firefly/renilla) }- \text { DMSO (firefly/renilla) }}\right]$ $\times 100 \%$

The $\mathrm{IC}_{50}$ values and curve fitting analyses were calculated with a Graphpad prism 5.

Molecular Docking Molecular docking was performed using the program eHiTS v12 from SimBioSys Inc. (Toronto, Canada). ${ }^{38,39)}$ eHiTS is an exhaustive flexible docking algorithm with a scoring function which incorporates both empirical and knowledge-based features. Open Babel (http:// openbabel.org) was used for manipulating the ligand chemical format and acquiring its 3D structure. PyMol (http://www. pymol.org/) was used for visual inspection of the results and the graphical representations.

The crystal structures of LXR $\alpha$ in a complex with the inhibitor GW3965 (PDB entry 3IPQ) and LXR $\beta$ with the inhibitor G58 (PDB entry 3L0E) in the Protein Data Bank was selected for the docking study. The eHiTS software package was used for flexible docking. Active site detection was carried out using the "-complex" parameter. The program automatically detected the ligand in the complex and selected the part of the target protein within a $7-\AA$ margin around the ligand as the active site. The compound was then docked into the active site using the highest accuracy mode of docking (" - accuracy" parameter set to 6). The scoring was according to the eHiTS_Score that is included in the eHiTS software package. We selected the compound with the best score and estimated the detailed binding patterns.
Acknowledgments This work was supported by New Drug Innovation 2009X09313-006 from the Ministry of Science and Technology of P.R. China. The authors are thankful to Shanghai ChemPartner Co., Ltd. and Shanghai PharmoGo Co., Ltd. for experimental assistance.

Conflict of Interest The authors declare no conflict of interest.

\section{References}

1) Pascual-García M., Valledor A. F., Arch. Immunol. Ther. Exp., 60, 235-249 (2012).

2) Mangelsdorf D. J., Thummel C., Beato M., Herrlich P., Schütz G., Umesono K., Blumberg B., Kastner P., Mark M., Chambon P., Evans R. M., Cell, 83, 835-839 (1995).

3) Janowski B. A., Grogan M. J., Jones S. A., Wisely G. B., Kliewer S. A., Corey E. J., Mangelsdorf D. J., Proc. Natl. Acad. Sci. U.S.A., 96, 266-271 (1999).

4) Yang C., McDonald J. G., Patel A., Zhang Y., Umetani M., Xu F., Westover E. J., Covey D. F., Mangelsdorf D. J., Cohen J. C., Hobbs H. H., J. Biol. Chem., 281, 27816-27826 (2006).

5) Wójcicka G., Jamroz W. A., Horoszewicz K., Bełtowski J., Postepy Hig. Med. Dosw., 61, 736-739 (2007).

6) Porkkala-Sarataho E., Salonen J. T., Nyyssonen K., Kaikkonen J., Salonen R., Ristonmaa U., Diczfalusy U., Brigelius-Flohe R., Loft S., Poulsen H. E., Arterioscler. Thromb. Vasc. Biol., 20, 2087-2093 (2000).

7) Zhao C., Dahlman-Wright K., J. Endocrinol., 204, 233-240 (2010).

8) Faulds M. H., Zhao C., Dahlman-Wright K., Curr. Opin. Pharmacol., 10, 692-697 (2010).

9) Huang C., J. Intern. Med., 12, 76-85 (2014).

10) Viennois E., Mouzat K., Dufour J., Morel L., Lobaccaro J. M., Baron S., Mol. Cell. Endocrinol., 351, 129-141 (2012).

11) Schultz J. R., Tu H., Luk A., Repa J. J., Medina J. C., Li L., Schwendner S., Wang S., Thoolen M., Mangelsdorf D. J., Lustig K. D., Shan B., Genes Dev., 14, 2831-2838 (2000).

12) Mitro N., Mak P. A., Vargas L., Godio C., Hampton E., Molteni V., Kreusch A., Saez E., Nature (London), 445, 219-223 (2007).

13) Dodo K., Aoyama A., Noguchi-Yachide T., Makishima M., Miyachi H., Hashimoto Y., Bioorg. Med. Chem., 16, $4272-4285$ (2008).

14) Hong C., Tontonoz P., Nat. Rev. Drug Discov., 13, $433-444$ (2014).

15) Calkin A. C., Tontonoz P., Nat. Rev. Mol. Cell Biol., 13, 213-224 (2012).

16) Collins J. L., Fivush A. M., Watson M. A., Galardi C. M., Lewis M. C., Moore L. B., Parks D. J., Wilson J. G., Tippin T. K., Binz J. G., Plunket K. D., Morgan D. G., Beaudet E. J., Whitney K. D., Kliewer S. A., Willson T. M., J. Med. Chem., 45, 1963-1966 (2002).

17) Shenoy S. D., Spencer T. A., Mercer-Haines N. A., Alipour M., Gargano M. D., Runge-Morris M., Kocarek T. A., Drug Metab. Dispos., 32, 66-71 (2004).

18) Houck K. A., Borchert K. M., Hepler C. D., Thomas J. S., Bramlett K. S., Michael L. F., Burris T. P., Mol. Genet. Metab., 83, 184-187 (2004).

19) Katz A., Udata C., Ott E., Hickey L., Burczynski M. E., Burghart P., Vesterqvist O., Meng X., J. Clin. Pharmacol., 49, 643-649 (2009).

20) Quinet E. M., Basso M. D., Halpern A. R., Yates D. W., Steffan R. J., Clerin V., Resmini C., Keith J. C., Berrodin T. J., Feingold I., Zhong W., Hartman H. B., Evans M. J., Gardell S. J., DiBlasioSmith E., Mounts W. M., LaVallie E. R., Wrobel J., Nambi P., Vlasuk G. P., J. Lipid Res., 50, 2358-2370 (2009).

21) Loren J., Huang Z., Laffitte B. A., Molteni V., Expert Opin. Ther. Pat., 23, 1317-1335 (2013).

22) Tamehiro N., Sato Y., Suzuki T., Hashimoto T., Asakawa Y., Yokoyama S., Kawanishi T., Ohno Y., Inoue K., Nagao T., Nishimaki- 
Mogami T., FEBS Lett., 579, 5299-5304 (2005).

23) Goldwasser J., Cohen P. Y., Yang E., Balaguer P., Yarmush M. L., Nahmias Y., PLoS ONE, 5, e12399 (2010).

24) González-Granillo M., Steffensen K. R., Granados O., Torres N., Korach-André M., Ortíz V., Aguilar-Salinas C., Jakobsson T., DíazVillaseñor A., Loza-Valdes A., Hernandez-Pando R., Gustafsson J. Å., Tovar A. R., Diabetologia, 55, 2469-2478 (2012).

25) Hoang M. H., Jia Y., Jun H. J., Lee J. H., Hwang K. Y., Choi D. W., Um S. J., Lee B. Y., You S. G., Lee S. J., Mol. Nutr. Food Res., 56, 900-911 (2012).

26) Sheng X., Wang M., Lu M., Xi B., Sheng H., Zang Y. Q., Am. J. Physiol. Endocrinol. Metab., 300, E886-E893 (2011).

27) Griffett K., Solt L. A., El-Gendy B. E.-D. M., Kamenecka T. M., Burris T. P., ACS Chem. Biol., 8, 559-567 (2013).

28) Aoyama A., Endo-Umeda K., Kishida K., Ohgane K., NoguchiYachide T., Aoyama H., Ishikawa M., Miyachi H., Makishima M., Hashimoto Y., J. Med. Chem., 55, 7360-7377 (2012).

29) Menke J. G., Macnaul N. S., Hayes N. S., Baffic J., Chao Y. S., Elbrecht A., Kelly L. J., Lam M. H., Schmidt A., Sahoo S., Wang J., Wright S. D., Xin P., Zhou G., Moller D. E., Sparrow C. P., Endocrinology, 143, 2548-2558 (2002).

30) Temple C. Jr., Wheeler G. P., Elliott R. D., Rose J. D., Kussner C. L., Comber R. N., Montgomery J. A., J. Med. Chem., 25, 1045-1050
(1982).

31) Breslin H. J., Diamond C. J., Kavash R. W., Cai C., Dyatkin A. B., Miskowski T. A., Zhang S. P., Wade P. R., Hornby P. J., He W., Bioorg. Med. Chem. Lett., 22, 4869-4872 (2012).

32) Le G., Vandegraaff N., Rhodes D. I., Jones E. D., Coates J. A., Thienthong N., Winfield L. J., Lu L., Li X., Yu C., Feng X., Deadman J. J., Bioorg. Med. Chem. Lett., 20, 5909-5912 (2010).

33) Chen J., Liu D., Butt N., Li C., Fan D., Liu Y., Zhang W., Angew. Chem. Int. Ed. Engl., 52, 11632-11636 (2013).

34) Rouf A., Aga M. A., Kumar B., Taneja S. C., Tetrahedron Lett., 54, 6420-6422 (2013).

35) Kim M. K., Lee H. S., Kim S., Cho S. Y., Roth B. L., Chong Y., Choo H., Bioorg. Med. Chem., 20, 1139-1148 (2012).

36) Khan M. F., Kumar P., Pandey J., Srivastava A. K., Tamrakar A. K., Maurya R., Bioorg. Med. Chem. Lett., 22, 4636-4639 (2012).

37) https://www.eurofinspanlabs.com/Catalog/Products/ProductDetails. aspx?prodId=uU1gN1SuSeY\%3d\&path=7,169\&leaf=7,169\&track = Add\%2f $\% 2$ fTarget+Class\%2f Nuclear+Receptor\#.

38) Zsoldos Z., Reid D., Simon A., Sadjad B. S., Johnson A. P., Curr. Protein Pept. Sci., 7, 421-435 (2006).

39) Zsoldos Z., Reid D., Simon A., Sadjad B. S., Johnson A. P., J. Mol. Graph. Model., 26, 198-212 (2007). 